\title{
Kindesunterhaltsrecht und Sozialleistungen - Schnittstellen, Verwerfungen, Probleme und Herausforderungen
}

Kindesunterhaltsrecht, Steuerrecht und Sozialleistungsrecht wirken zusammen mit dem Ziel der Sicherung der Unterhaltssicherung von Kindern, aber sie weisen auch wichtige Unterschiede auf (so verfolgt etwa das Unterhaltsrecht das Ziel der Deckung des angemessenen Bedarfs, während das Sozialgeld des SGB II das Ziel der Grundsicherung verfolgt $)^{1}$. Dabei kommt es in vielen Bereichen zu Verwerfungen; die unzureichende Koordination zwischen Familienrecht, Steuerrecht und Sozialrecht ist bereits häufig kritisiert worden (so auch vom Bundesverfassungsgericht $2003^{2}$ und vom Bundestag 2000 3 ). Empirische Untersuchungen haben nachgewiesen, dass für Kinder getrennt lebender Eltern in der Mehrzahl der Fälle die Kindesunterhaltszahlungen des barunterhaltspflichtigen Elternteils deutlich unter dem Existenzminimum des Kindes liegen. ${ }^{4}$ Die prekäre Einkommenssituation vieler alleinerziehender Eltern ${ }^{5}$ sowie von $\mathrm{Fa}$ milien mit mehreren Kindern im unteren Einkommensbereich werfen ebenfalls die Frage auf, wie Kinder und diese Familien besser unterstützt werden können.

Die Auseinandersetzung mit unterschiedlichen Veränderungsvorschlägen in diesem Schnittbereich ist daher eine wichtige Aufgabe der Kommission.

\section{Erhöhung von Kindesunterhaltsbeträgen?}

Vorgeschlagen wird die Erhöhung des Mindestunterhalts des $\mathbb{S} 1612$ a BGB durch eine veränderte Berechnung des steuerlichen Kinderfreibetrags und Einbeziehung der Bildungs- und Ausbildungskosten. ${ }^{6}$ Vorgeschlagen wird weiterhin, dass der Mindestunterhalt höher angesetzt werden sollte als das sächliche Existenzminimum nach dem Steuerrecht, denn auch das unterhaltsrechtliche Existenzminimum, der Selbstbehalt der oder des Unterhaltsverpflichteten, sei etwa 30 Prozent höher als sein steuerliches Existenzminimum oder sein SGB II-Grundsicherungsbedarf. ${ }^{7}$

\section{Effizientere Rechtsdurchsetzung und Beratung sowie verbesserte Regressmöglichkeiten?}

Die Beistandschaft des Jugendamts spielt eine wichtige Rolle für die Rechtsdurchsetzung von Kindesunterhaltsansprüchen gegen den barunterhaltspflichtigen Elternteil. Sie könnte durch mehr Personalmittel für Jugendämter und verbesserte
Schulungen effizienter gestaltet werden. Auch die Durchsetzung von Regressansprüchen gegen Unterhaltsschuldner könnte verbessert werden. Die Rechnungshöfe haben bereits häufig die geringe Rückholquote kritisiert.

Zur effizienteren Ausgestaltung des Regresses von Sozialleistungsträgern gegen Barunterhaltspflichtige wird vorgeschlagen, die Erweiterungen der Informationspflichten über $\mathbb{S} 98$ SGB X hinaus ebenso wie die Möglichkeiten der Abzweigung ( $\sqrt{ } 48 f$ SGB I) zu nutzen, um den Ertrag aus Rückgriffsansprüchen zu steigern oder den Rückgriff entbehrlich zu machen. ${ }^{8}$ Flügge ${ }^{9}$ schlägt vor, dass Unterhaltsvorschusskassen in stärkerem Maße als Inkassoagenturen für Kindesunterhaltsansprüche und auch Betreuungsunterhaltsansprüche tätig werden sollten, um die Effizienz zu steigern und die individuelle Belastung mit der Rechtsdurchsetzung zu vermindern.

Erhöhung der Kinderregelsätze des SGB II und Einführung eines eigenen Verfahrens zur Bedarfsermittlung von Kindern

Diese Problematik hat durch die Vorlagebeschlüsse des Bundessozialgerichts vom 27. Januar 200910

1 Vgl. ausführlich dazu Scheiwe, Kindesunterhalt und Sozialleistungen, in: Kinder und Jugendliche im Sozialleistungssystem. Bundestagung des Deutschen Sozialrechtsverbandes 2008, Berlin 2009, S. 51-77.

2 BVerfGE 108, 52ff. = FamRZ 2003, 1370, 1374.

3 BT-Drs. 14/3781.

4 Forsa, Unterhaltszahlungen für minderjährige Kinder in Deutschland, 2002.

5 Nach den jüngsten Mikrozensus-Daten von 2007 lag der Anteil Alleinerziehender, die ALG II-Leistungen für sich oder Sozialgeld für die Kinder beziehen, bei 41 Prozent. Wenn öffentliche Sozialleistungen und private Unterhaltszahlungen zum Erwerbseinkommen Alleinerziehender hinzu gerechnet wurden, hatten immer noch 11 Prozent der betreuenden Elternteile im Westen und 17 Prozent im Osten monatlich weniger als 900 Euro zur Verfügung Forsa (FN 4), S.151.

6 So etwa Breithaupt in der Sachverständigenanhörung zum UÄndG (Deutscher Bundestag, Rechtsausschuss, Protokoll Nr. 28, Sitzung vom 16.10.2006, unter www. bmj.bund.de).

7 M. Diwell in der Sachverständigenanhörung zum UÄndG, ebd.

8 Nachweise bei Eichenhofer, Gutachten B zum 64. Deutschen Juristentag, 2002, S. B 55

9 Flügge, Die ungelöste Frauenfrage: Was wird aus dem Familienernährer?, in: Scheiwe (Hrsg.) Soziale Sicherungsmodelle revisited, 2007, S. 185 ff. 194.

10 Beschluss des 14. Senats des BSG vom 27.1.2009 (Az. B 14/11b AS 9/07 R und B 14 AS 5/O8 R).
Prof. Dr.

Kirsten Scheiwe

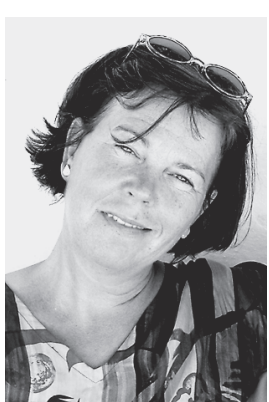

Mitglied der Kommis-

sion Recht der sozialen Sicherung, Familienlastenausgleich des djb; Universität Hildesheim 
und des Landessozialgerichts Hessen vom 29. Oktober 2008

11 an das Bundesverfassungsgericht an rechtlicher Brisanz gewonnen. Das Bundesverfassungsgericht befasst sich nun mit der Frage, ob die Regelung der Kinderregelsätze des $\mathbb{} 28$ Abs. 1 S. 3 Nr. 1 SGB II verfassungsgemäß ist. Bereits zuvor wurde in der öffentlichen Diskussion vorgeschlagen, die Regelsätze des SGB II für Kinder und Jugendliche (derzeit 211 Euro für 0- bis 13-Jährige und 281 Euro für 14- bis 17-Jährige) zu erhöhen und die Altersgruppeneinteilung des SGB II zu verändern, die auch von der Altersgruppenstaffelung der familiengerichtlichen Unterhaltstabellen abweicht. Der Kinderregelsatz sollte in einem eigenen Verfahren selbständig berechnet werden auf der Grundlage entsprechender Erhebungen zum Bedarf von Kindern und auch die Kosten für Bildung berücksichtigen. Verschiedene Studien haben dazu detaillierte Vorschläge unterbreitet ${ }^{12}$, die deutliche Anhebungen fordern. Um eine flexiblere Möglichkeit zur sozialrechtlichen Gewährung von einzelfallbezogenen Leistungen als Teil des Grundsicherungsbedarfs des SGB II zu ermöglichen, wird die Einführung einer Härtefallklausel vorgeschlagen.

\section{Sach- und Dienstleistungen für Kinder erweitern, vor allem im Bildungsbereich}

Ein Schritt in diese Richtung ist die Einführung eines Rechtsanspruchs auf Förderung in einer Kindertageseinrichtung oder Tagespflege für Kinder ab einem Jahr ab 2013 durch das Kinderförderungsgesetz (KiFöG). ${ }^{13}$ Nachdem das SGB II die einmaligen Leistungen fast ganz beseitigt hat, sind Lehrbücher und Lernmaterial zum Schuljahrsbeginn ein besonderes Problem. Das ,Schulstarterpaket' soll hier Hilfe leisten, weist aber zahlreiche Schwächen auf, die in der Sachverständigenanhörung von einem Mitglied der Kommission kritisiert wurden. ${ }^{14}$ Leistungen, die von den Ländern und Kommunen zu gewähren sind bzw. teilweise bereits gewährt werden, sind Lehrmittelfreiheit, Zuschüsse zu den Essenskosten an Ganztagsschulen, Angebote von schulischer Nachhilfe und Sprachförderung oder die Gewährung von kostenlosem Schülertransport auch in höheren Schulklassen oder die kostenlose Nutzung des öffentlichen Nahverkehrs.

\section{Reform von Kindergeld/Kinderfreibetrag und Einführung eines einkommensabhängigen Kindergeldzuschlags}

Der Kinderfreibetrag stellt das Existenzminimum des Kindes frei und dient der Herstellung der horizontalen Steuergerechtigkeit. Nur wenn das Kindergeld höher ist als die steuerliche Entlastungswirkung, ist es eine Förderleistung. Die steuerliche Komponente könnte auch durch Einarbeitung des Kinderfreibetrags als Grundfreibetrag geschehen. Sinnvoll wäre daneben ein Kindergeld als echte Förderleistung, das mehr gewährleistet als die Steuerfreiheit des Existenzminimums der Familie. ${ }^{15}$ Diskutiert wird auch die Gewährung eines einkommensabhängigen Kindergeldes oder Kindergeldzuschlags, welches den bisherigen Kinderzuschlag sowie das Sozialgeld nach SGB II ersetzen könnte.

\section{Verbesserung der Einkommenssituation von Erwerbstätigen mit Niedrigeinkommen}

Darauf zielen Vorschläge wie etwa gesetzlich geregelte Mindestlöhne $a b$, um es angesichts der zunehmenden Lohnspreizung und der Zunahme prekärer Beschäftigungsverhältnisse den Unterhaltspflichtigen zu erleichtern, ihre Unterhaltspflichten zu erfüllen. Dadurch könnten auch erwerbstätige Alleinerziehende gezielt gefördert werden.

Eine andere Möglichkeit wäre es, die Erwerbstätigenfreibeträge (\$ 30 II SGB II) und Hinzuverdienstgrenzen zum SGB II zu erhöhen ${ }^{16}$ und sich dabei etwa an den früheren Freibeträgen bei der Arbeitslosenhilfe zu orientieren (\$194 SGB III i.d.F. bis Ende 2003) oder zumindest an den Freibeträgen in der Haushaltsgemeinschaft ( $\mathbb{9}$ Abs. 5 SGB II) oder den Freibeträgen bei übergeleitetem Unterhalt ( $\mathbb{S} 33$ SGB II). ${ }^{17}$

\section{Möglichkeiten der Verbesserung der Einkommenssituation Alleinerziehender}

Die verstärkte Müttererwerbstätigkeit wird als ein wesentliches Mittel betrachtet, um Kinderarmut zu vermindern - befürchtet wird demgegenüber, dass der Druck zur Ausweitung der Erwerbsarbeit auf Kosten der Kinder gehe und die Mehrfachbelastung der Mütter sich noch verstärke. Die Veränderungen der Rangregelungen von Unterhaltsansprüchen im UÄndG durch Verweisung von betreuenden Elternteilen auf den zweiten Rang gehen ebenfalls in die Richtung, die Müttererwerbstätigkeit weiter zu verstärken.

Im SGB II ist eine Erwerbstätigkeit dann zumutbar, wenn ein Kind drei Jahre alt und die Kinderbetreuung gesichert ist. Von einigen wird darüber hinaus auch die Ausweitung der Erwerbsobliegenheit auf Mütter von Kindern unter drei Jahren gefordert, um die Gefahr zu vermindern, sich von der Erwerbsarbeit zu entfernen, und um Eingliederungsprobleme in

11 Entscheidung des 6. Senats des LSG Hessen vom 29.10.2008 (Az. L 6 AS 336/07).

12 Z.B. Martens, Rudolf (2008): Zur Bestimmung eines bedarfsgerechten Existenzminimums für Kinder nach dem Statistikmodell gemäß § 28 SGB XII (Sozialhilfe). - In: Was Kinder brauchen ... Für eine offene Diskussion über das Existenzminimum für Kinder nach dem Statistikmodell gemäß § 28 SGB XII (Sozialhilfe), Der PARITÄTISCHE Gesamtverband, Berlin.

13 Gesetz zur Förderung von Kindern unter drei Jahren in Tageseinrichtungen und in der Kindertagespflege (Kinderförderungsgesetz - KiföG) vom 10.12.2008 BGBI. I S. 2403.

14 Entwurf eines Gesetzes zur Förderung von Familien und haushaltsnahen Dienstleistungen (Familienleistungsgesetz - FamLeistG), BTDrs. 16/10809. Stellungnahme, Sachverständigenanhörung. Vgl. dazu die Stellungnahme der Kommissionsmitglieder Dr. C. Fuchsloch und U. Spangenberg für den djb vom 21.11.2008 anlässlich der Anhörung im Finanzausschuss des Deutschen Bundestages am Montag, den 24. November 2008 zum Gesetzentwurf des FamLeistG - BT-Drs. 16/10809 - sowie zur Stellungnahme des Bundesrates - BR-Drucksache 753/08 unter www.djb.de/Kommissionen/ kommission-recht-der-sozialen-sicherung-familienlastenausgleich/ St_08-21/ sowie die Stellungnahme in der Ausschussanhörung von U. Spangenberg.

15 Wissenschaftlicher Beirat für Familienfragen, Gerechtigkeit für Familien, 2001, S. 80.

16 Scheiwe, Existenzsicherung zwischen Sozial- und Familienrecht in der BRD, in: Scheiwe (FN 72), S. 112.

17 Spindler, Abhängig oder unabhängig?, in: Scheiwe (ebd.), S. 93. 
den Arbeitsmarkt zu vermindern. ${ }^{18}$ Diese Vorschläge sind problematisch. Bereits jetzt sind die Erwerbsquoten von alleinerziehenden Müttern höher als die von verheirateten Müttern, aber das Erwerbseinkommen ist häufig relativ niedrig. Insbesondere für erwerbstätige Alleinerziehende sind ergänzende Sozialleistungen sinnvoll; der Kinderzuschlag ist hier bisher nicht zielführend. Außerdem sollten Beiträge für eine ausreichende Altersvorsorge in höherem Umfang von Erwerbseinkommen abzugsfähig sein als bisher. Vermittlung in geringfü- gige Beschäftigungsverhältnisse eröffnet jedoch keine sinnvolle langfristige Perspektive, ist in der Praxis der Jobcenter für Mütter junger Kinder jedoch häufig. Deshalb wäre es empfehlenswert, für ,Berufsrückkehrerinnen' wieder Rechtsansprüche auf Qualifizierungs- und Umschulungsmaßnahmen einzuführen, die jedoch durch Hartz III eingeschränkt und durch Ermessensleistungen ersetzt wurden.

18 So etwa Eichenhofer (FN 8), S. B 38.

\section{Finanzmarktkrise, Föderalismusreform und die Folgen - ein Ausblick}

Bund, Länder und Gemeinden müssen laut der Steuerschätzung vom Mai 2009 bis 2013 mit über 300 Milliarden Euro weniger auskommen als noch im November 2008 angenommen. Allein für 2009 wird erwartet, dass die Steuereinnahmen um 45 Milliarden Euro niedriger ausfallen. Gleichzeitig steigen die Kosten: Höhere Arbeitslosigkeit führt nicht nur dazu, dass weniger Menschen in die Sozialversicherung einzahlen; es werden auch mehr Ansprüche geltend gemacht. Das Kurzarbeitergeld belastet den Haushalt der Bundesagentur für Arbeit (BA) zusätzlich. Die BA rechnet damit, dass sie in diesem Jahr 3,4 Milliarden Euro für die Kurzarbeit benötigen wird, sagte BA-Vorstandsmitglied Raimund Becker im Juni der „Süddeutschen Zeitung“. Dies ist über eine Milliarde mehr als geplant. Es ist somit absehbar, dass die Bundesagentur höhere finanzielle Unterstützung aus dem Bundeshalt benötigt. Hinzu kommen die Maßnahmen der Konjunkturpakete auf Bundes- und Länderebene, die ebenfalls finanziert werden müssen, der „Schutzschild“ für die Banken und andere Stabilisierungsmaßnahmen.

Zudem hat die Kommission von Bundesrat und Bundestag zur Modernisierung der Bund-LänderFinanzbeziehungen (Föderalismuskommission II) im März dieses Jahres die Einführung einer Schuldenbegrenzungsregelung beschlossen. Diese wurde mit dem anschließenden Gesetzgebungsverfahren, das am 12. Juni 2009 im Bundesrat abgeschlossen worden ist, im Grundgesetz verankert. Die Schuldengrenze tritt für den Bund 2016, für die Länder 2020 in Kraft. Konnten Bund und Länder bisher in der Höhe, in der sie Investitionen tätigten, Kredite aufnehmen, ist dieser Weg dann weitgehend verschlossen. Die neue Regelung begrenzt die Möglichkeit einer strukturellen Neuverschuldung - also einer solchen, die nicht zurückgeführt werden muss - für den Bund auf 0,35 Prozent des Bruttoinlandsproduktes, was derzeit etwa neun Milliarden Euro im Jahr entspricht. Für die Länder ist eine strukturelle Verschuldung dann gar nicht mehr möglich. Zulässig ist ab Inkrafttreten für die Länder nur noch eine sogenannte konjunkturelle Verschuldung, mit der auf konjunkturelle Schwankungen reagiert werden kann. Diese hat aber nahe der Null zu bleiben und muss innerhalb eines angemessenen Zeitraumes zurückgeführt werden. In Notsituationen (Naturkatastrophen, aber auch einer der jetzigen vergleichbaren Finanzmarktkrise) ist eine Verschuldung weiterhin erlaubt, jedoch gekoppelt an einen Tilgungsplan - auch hier also mit Rückführungsverpflichtung. Konjunkturelle Verschuldungsmöglichkeiten und Notfallverschuldung sind auch für den Bund möglich.

Auch wenn bis zum eigentlichen Inkrafttreten noch einige Jahre vergehen - Auswirkungen sind bereits in naher Zukunft zu erwarten, denn bis 2016 bzw. 2020 müssen der Bund und die Länder erreichen, dass sie diese Vorgaben einhalten können. Es ist also davon auszugehen, dass sie in den nächsten Jahren finanzpolitisch einen harten Konsolidierungskurs fahren, dies bei einer voraussichtlich auch noch mittelfristig schwachen Wirtschaftsentwicklung mit entsprechend geringen Steuereinnahmen. Dieser Weg ist bei einer Neuverschuldung, die Bundesfinanzminister Peer Steinbrück für dieses Jahr mit 80 Milliarden Euro für den Bundeshaushalt beziffert, noch sehr lang, nicht zuletzt, weil man dies mit den mittelfristig niedrigeren Steuereinnahmen zusammen sehen muss.

Gerade in Zeiten leerer Kassen ist eine Diskussion über eine weitere Privatisierung von Risiken sei es bei der Rente, der Krankenversicherung oder

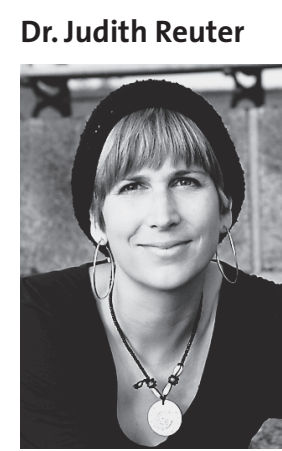

Mitglied der Kommission Recht der sozialen Sicherung, Familienlastenausgleich des djb; Referentin für Bund-Länder-Finanzbeziehungen und Föderalismusreform, Senatskanzlei, Hamburg 